\title{
Modeling a Dynamic Supply Chain Management System for an Utility Company in Nigeria
}

\section{Moses Okechukwu Onyesolu1, Josiah Chukwuemeka Abara², Chiamaka Ijeoma Chukwuneke1, Doris Chinedu Asogwa ${ }^{1}$}

\author{
${ }^{1}$ Department of Computer Science, Nnamdi Azikiwe University, Awka, Nigeria \\ ${ }^{2}$ Department of Physics/Electronics, Federal Polytechnic, Owerri, Nigeria \\ Email: mo.onyesolu@unizik.edu.ng, abarajosiah84@gmail.com, ci.chukwuneke@unizikedu.ng, dc.sogwa@unizik.edu.ng
}

How to cite this paper: Onyesolu, M.O., Abara, J.C., Chukwuneke, C.I. and Asogwa, D.C. (2018) Modeling a Dynamic Supply Chain Management System for an Utility Company in Nigeria. Journal of Software Engineering and Applications, 11, 275-284. https://doi.org/10.4236/jsea.2018.116017

Received: May 1, 2018

Accepted: June 8, 2018

Published: June 12, 2018

Copyright (c) 2018 by authors and Scientific Research Publishing Inc. This work is licensed under the Creative Commons Attribution International License (CC BY 4.0).

http://creativecommons.org/licenses/by/4.0/

(c) (i) Open Access

\begin{abstract}
Service and repair companies, big stores, industries and power companies having complex issues on inventory brought this idea for solution. The inventory department of PHCN generating unit has obsolete and sometimes non-availability of materials. The company faces maintenance, repairs, replacement demands and outage-related problems. In order to cater for these demands, some classifications that would aid PHCN in making effective decisions as regards spare or service parts were made. This research has developed inventory control system that provides automated inventory management system and graphical interface ability for decision making. This is to "right-size" inventories such that the total level of inventory held can be reduced and replenished without exposing the company to risk of stock-out. The system is a model for dynamic supply chain management using spare parts inventory policy tools to enable PHCN to dynamically perform continuous review, one to one lot, service differentiation, backordering, demand lead time, threshold rationing and clearing mechanism.
\end{abstract}

\section{Keywords}

Inventory Management, Lead Time, Maintenance, Replacement, Stochastic Demand

\section{Introduction}

Customers are more demanding, requiring greater choice, quality, value for money and timely delivery. This implies greater concern for inventory control on the part of companies and service providers. Inventory control is based on acquiring, storing and managing the inventory in such a manner that stock is 
always available to cater for contingencies, maximize profit and minimize wastage, and avoid disservice to customers. However, inventory levels are affected by customer service expectations, demand uncertainty, and the flexibility of the supply chain, which employing strategies to obtain optimal balance between these three extremes makes for a better company in terms of reduction in disservice and customer dissatisfaction [1]. PHCN currently holds in excess parts inventory used to service the generation facilities. The ways of handling its inventory has failed to cope with factors like stochastic demands, better service levels, and shorter lead times and providing perfect heuristics for inventory-related decision making [1].

Therefore it is imperative to accurately forecast spare parts requirements and to optimize existing inventory policies using significant decision support. The complex factors that enhanced the criticality of spare parts in companies can be expressed in questions such as: Are demands classified? What is the resultant effect of backorders on the demand classes? Are the customers differentiated? Are the demands and replenishment lead times stochastic or deterministic? And ultimately what does the company stand to gain, with the development of such models? The practical application of adopting a coordinated model-driven decision support approach for spare parts inventory management and control throughout the entire supply chain has the potential to simultaneously reduce service parts inventory levels and improve parts availability at all times [2]. In strong terms, the model was seen to be implemented in power generation, transmission, and distribution companies and other industries to serve the following purposes: To curb the incessant power outages by managing the inventory in a way that repair, replacement and maintenance demands are met. To have the ability to check the criticality of spare parts with the service level expected by a particular demand class (gold, silver, bronze) as well as the average number of backorder and the fill rate of those demands.

The operating realities of Power Holding Company of Nigeria (PHCN) informed this work. The focus is to provide continuous review for service differentiated demand classes and threshold clearing mechanism using a discrete event simulation. Note also that the service differentiation here involves three demand classes which are classified as gold, silver and bronze. The study did not employ mathematical/analytical approach rather it uses the simulation approach to build a decision support model. The scope of this study did not include the analysis of costs i.e. analyzing the carrying, holding and stock-out costs. The simulation approach employed in this study is used to check the average number of backorders and the fill rate for demand classes.

\section{Background Information}

PHCN is charged with the primary role of generating, transmitting, distributing and marketing electricity in Nigeria at a regulated and un-privatized environment. The company operates a maintenance-like environment which focuses on 
providing constant support for the operation of a single unit, plant or component (or a fleet or group of components), and ensuring that operational requirements are achieved [3]. Plagued with many complications, the least is the broad range of products, which makes the problem of determining right-sized inventories more difficult and also the absence of an effective maintenance culture. Essentially, PHCN must maintain a balance between the plant availability and output against the electricity demand and the plant's capacity through a better understanding of spare parts demand. This would be of potential improvement because if the company can understand when it will need parts, then the company can plan accordingly to promote just-in-time delivery and minimal inventory [4].

The Nigerian power transmission network is characterized by prolonged and frequent outages, outages like planned outages and forced outages [4] which can be associated with aging equipment/defects (leading to frequent conductor/jumper cuts, frequent earth faults resulting from reduction in overhead clearance and refuse burning, circuit breaker problems), lightning, wind, birds/animals, vandalization, accidents and poor job execution by contractors. The study revealed that the existing transmission network is characterized by poor maintenance and over aged leading to the collapse of several spans; and that prolonged and frequent outages are phenomena in the transmission networks. Again, most of the transmission lines are very long and fragile leading to frequent conductor cuts which gives rise to high voltage drops and power losses in the network.

Batarda [5] proffered solutions which include carrying out a study to identify all weak areas in the network with a view to strengthen the network, carrying out planned and routine maintenance on the network to reduce the incident of collapsed spans, others include addition of more substations into the network to assist in the reduction of long lines and improve the voltage profiles of the network, and promptly rectifying faults and energizing all the lines to reduce the incidence of vandalization. Arobieke et al. [6] and Samuel et al. [7] examined voltage collapse on the Nigerian National Grid. They maintained that voltage instability and collapse contribute to large extent system collapse or blackouts and it is one of the major concerns for today's electric power system operations. In furtherance, the Nigerian National Grid (NNG) experiences on an average thirty-five (35) system collapses every year over a ten (10)-year period from 2000 to 2009 [7]. Indeed, Table 1 showed an overview of system collapse for another ten (10)-year period from 2008 to 2017 [8].

From Table 1, Ogbuefi et al. [8] made some deductions which boiled down to the fact that the grid is facing both technical and political challenges. The technical challenges include: obsolete power system equipment, inadequate compensation equipment on the system, faulty and weak protection system, weak-network leading to indiscriminate tripping of the lines, generation shortage due to increasing consumers demand, the weak wheeling power and poor maintenance culture. The Political challenges are: neglect from Government 
Table 1. Classification of system collapse for the period 2009-2018 on the nigerian national grid (NNG) [8].

\begin{tabular}{ccccccccccc}
\hline Nature of Disturbance/Year & 2008 & 2009 & 2010 & 2011 & 2012 & 2013 & 2014 & 2015 & 2016 & 2017 \\
\hline Partial Collapse & 15 & 20 & 20 & 06 & 08 & 02 & 07 & 02 & 06 & 02 \\
Total Collapse & 25 & 19 & 22 & 13 & 16 & 22 & 02 & 10 & 04 & 12 \\
Total System Collapse & 40 & 39 & 42 & 19 & 24 & 24 & 09 & 12 & 10 & 14 \\
\hline
\end{tabular}

over long period, the lip service paid by the National Leaders to the development of the power sector and corruption. Ogbuefi et al. [8] finally opined that solving these challenges would to a great extent solve the power problems of the nation.

\section{Materials and Methods}

We developed and modeled a dynamic supply chain management system adopting a hybrid methodology. The methodology was derived from the combination of the Structured System Analysis and Design Methodology (SSADM) and the Simulation Project Life Cycle proposed by [9]. The investigative phase of the SSADM was deployed as the paradigm for systematic study in order to obtain information on the current trends in the research area of supply chain management. The information obtained necessitated the definition of a high-level model (HLM) for the system as shown in Figure 1. Simulation Project Life Cycle was employed to develop the decision support simulation model that will check the stochastic demand of spare parts and to optimize inventory so as to find the average number of backorders and fill rate. In addition, a composite stepwise 2-dimensional graphical representation which represented the concept model of the study was formulated to show state transitions of how backorder, stock level, number of bronze demand arrivals not due, number of silver demand arrivals not due and number of replenishment order on transit vary against time.

\section{System Design}

The system observed service differentiation with a threshold policy for rationing and for clearing backlogged demands. There are three classes of customers having different service level constraint for identical spare parts. We assumed inventory for an item held and replenished over time to fill reoccurring demand from three customer classes as $i=1,2,3$. We also assumed demand from class $i$ follows a Poisson process with rate $\lambda_{p}$ implying a total demand rate of $\lambda=\lambda_{1}+\lambda_{2}$ $+\lambda_{3}$ [10]. The demand arrival process results in service level agreement of checking outages (forced, planned, urgent and emergent) and corrective maintenance issues (repairs) [11]. The gold demand (GD) constitutes the highest demand class while the other two classes are demands caused by maintenance and other trivial emergent issues. They include preventive and elective maintenance orders i.e. silver demand (SD), the middle class and emergent issues i.e. bronze demand (BD) the lowest class. 


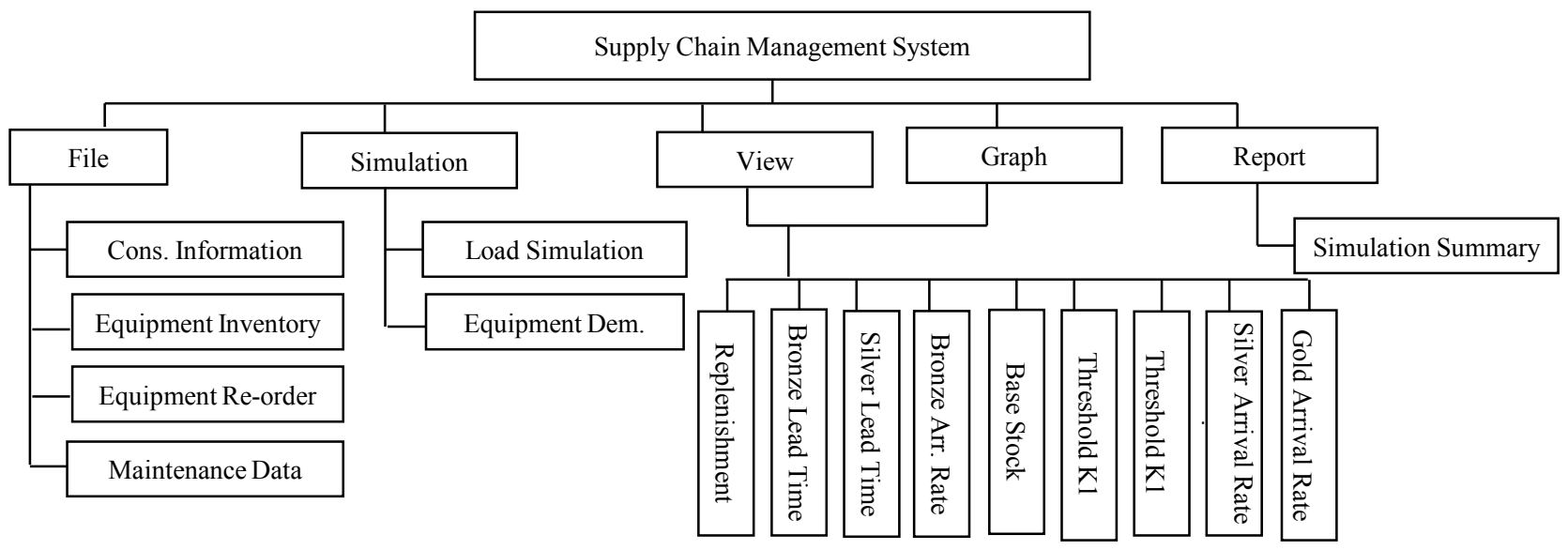

Figure 1. The high level model of the supply chain management system.

The highest consideration is given to the orders that come from outages and corrective maintenance (mostly repairs). This constitutes the most critical (or highest priority) gold class because not filling these orders might put the plant in an inoperative state for a very long time. The next is silver class that constitutes the medium priority and bronze class that constitute the least critical (or lowest priority).

All arrivals i.e. GD with arrival rate of $\lambda_{1}$, SD with arrival rate of $\lambda_{2}$ and $\mathrm{BD}$ with arrival rate $\lambda_{3}$ for each spare part are filled from the same pool of inventory which implies a total arrival rate of $\Sigma \lambda_{k}$ The stock is controlled by a continuous review threshold level policy, base stock policy with a base stock level S [12]. Demand lead time for all the demand arrivals differ. Here, all demand arrivals, whether GD, SD or BD triggers off a replenishment order which will arrive after $\mathrm{L}_{\mathrm{r}}$ time unit. The threshold policy employed worked as: Demands from three classes are filled on a FCFS basis as long as the on-hand inventory level is greater than or equal to $\mathrm{K}^{2}$ otherwise, bronze demand class is backlogged. Similarly, demands from gold and silver classes are filled on FCFS basis as long as on-hand inventory is greater than or equal to $\mathrm{K}^{1}$. Once the on-hand inventory falls below $\mathrm{K}^{1}$, the silver and bronze demand class is backlogged. Two policies were governed by a threshold level $\left(\mathrm{K}^{2}, \mathrm{~K}^{1}\right)$ in this study. That is, one for when a demand occurs (rationing policy) and one for when a unit of stock is delivered (backorder clearing mechanism).

\subsection{Threshold Rationing Policy}

1) PI $>$ K2: Satisfy all demands.

2) $\mathrm{PI}>\mathrm{K} 1$ and PI < K2: Satisfy Gold and Silver demands and backorder the Bronze demands.

3) PI < K1: Satisfy Gold Demands and backorder Silver and Bronze

4) $\mathrm{PI}=0$ : Backorder all classes of demands

\subsection{Threshold Backorder Clearing Mechanism}

1) On the arrival of replenishment order an existing backorder will be satisfied 
on first come first serve basis (FCFS), if one exists;

2) Otherwise the replenishment order is added to the physical inventory.

3) Here, clearing backorders allows for partial clearing (i.e. where a backorder is partially filled if the physical inventory cannot be enough to clear the total backorder)

Where PI is Physical Inventory, $\mathrm{K}^{2}$ is first threshold level; $\mathrm{K}^{1}$ is second threshold level [13].

\section{Results and Discussion}

The results of the simulation and modeling are shown in Figure 2 to Figure 6 and Table 2 to Table 3. Some of the implementation of the developed model is shown in Figure 2 to Figure 4, how the model is operated in addition to formulated concept model which $\mathrm{x}$-rays the composite stepwise 2-dimentional graphical representation of the stochastic behaviour of the system. Double click on the PHCN simulator icon, a flash window appears, the system is loaded quickly. Thereafter, a security dialogue box appears (Figure 2) requesting for login user name and password. This is to avoid unauthorized use of the system. On successful entry of the user name and password, the system launches to the main menu. The main menu displays a window with file, simulation, view, graph and report on the menu bar. Clicking on the simulation menu, the software launches into the simulation environment. Here, several simulation runs as well as sensitivity analysis can be performed.

The input dialogue box is the inventory simulator dialog box (Figure 3 ). The required input parameters for the simulation are keyed into the Inventory Simulator Dialog Box, in line with the dataset to be simulated [14]. The clear button is for clearing the inputted values when the need arises while the close button closes the dialogue box. The Start button prompts the computer to start simulation after data input on the dialog box which will trigger another display of the sensitivity analysis dialog box.

The setting in the sensitivity analysis dialog box help to select the command options desired. The setting is completely flexible. The parameter that is selected for sensitivity analysis in the dialog box is the threshold level $\mathrm{K}^{1}$, while the minimum and maximum iteration values are 1 and 2, respectively. The step wise value is 1 . At the threshold level $\mathrm{K}^{1}$, simulation is run and its outputs are displayed in the final simulation outputs. Similarly, any other sensitivity parameter can be chosen while any value inputted in the iteration range as step wise value.

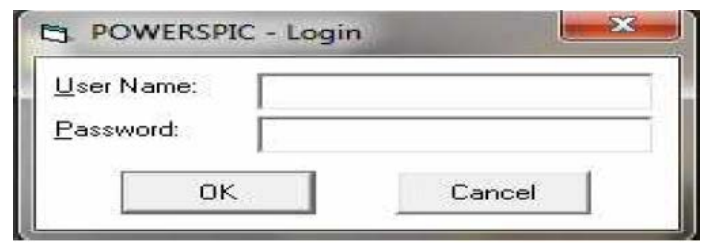

Figure 2. Inventory simulator dialog box. 


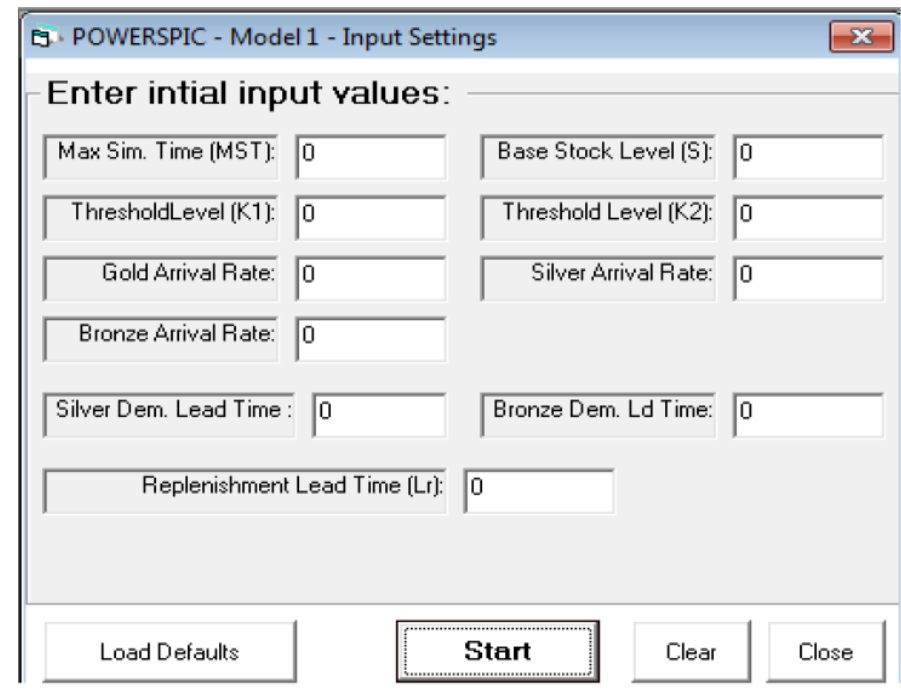

Figure 3. Inventory simulator dialog box.

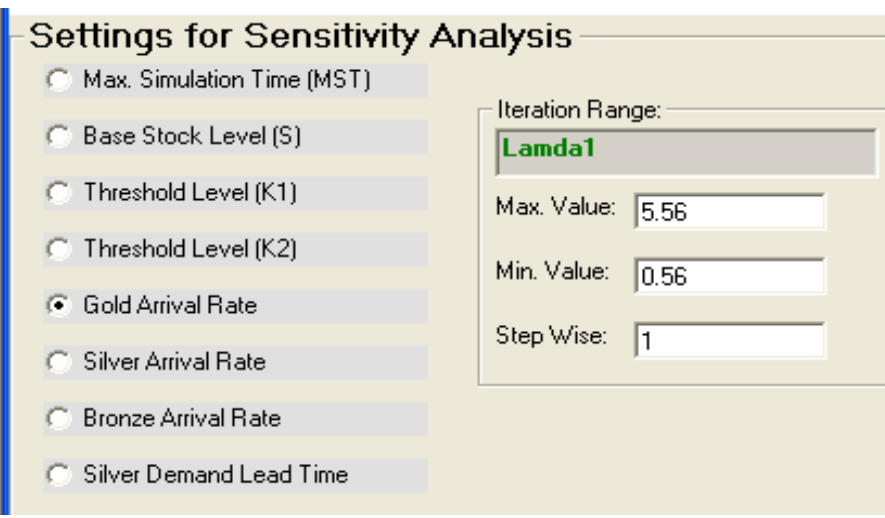

Figure 4. Sensitivity analysis dialog box.

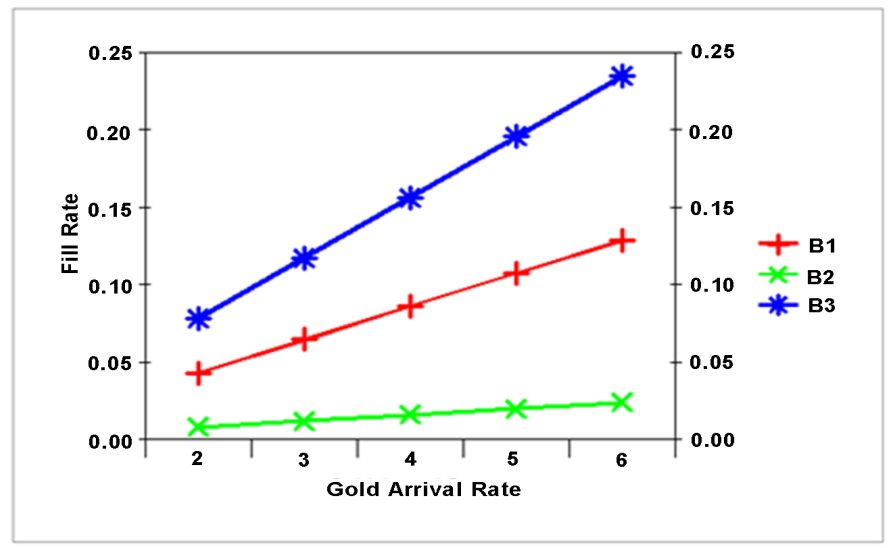

Figure 5. Fill rates results on gold arrival rate sensitivity analysis.

After setting the values for the sensitivity analysis, the start button is clicked from the inventory simulator dialog box. When simulation run for a step increment has been completed, output result generated for each perturbation is registered in the final simulation output which displays B1, B2, B3, ANB1, ANB2 and 


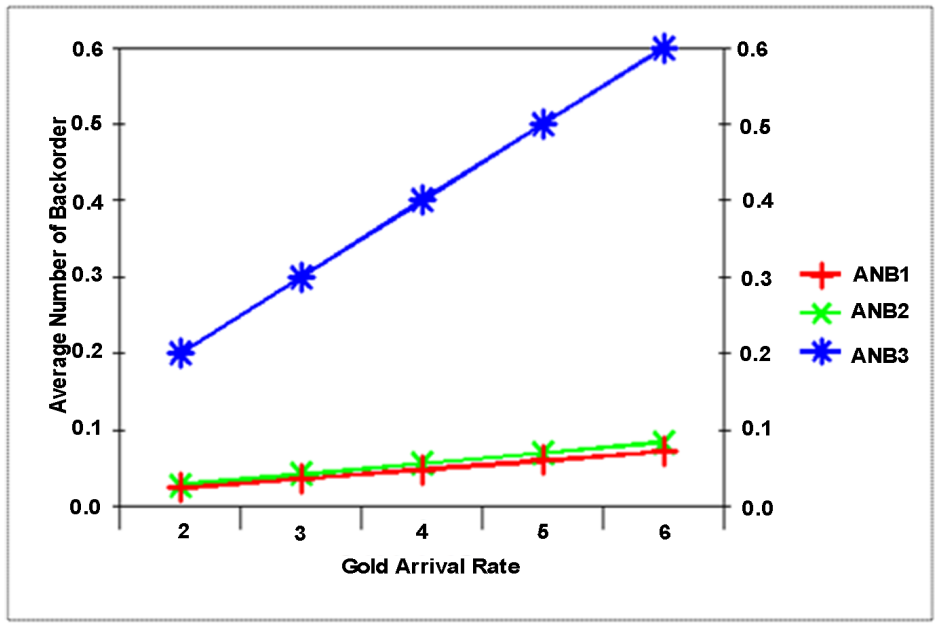

Figure 6. Average number of backorder (ANB) results on gold arrival rate sensitivity analysis.

Table 2. Fill Rates on the Gold Arrival Rate Sensitivity Analysis. Dataset [MST $=6, S=10$, $\left.\mathrm{K}^{2}=5, \mathrm{~K}^{1}=2, \lambda_{2}=4, \lambda_{3}=3, \mathrm{SL}_{\mathrm{d}}=1, \mathrm{BL}_{\mathrm{d}}=0.12, \mathrm{~L}_{\mathrm{r}}=1\right]$.

\begin{tabular}{cccc}
\hline$\beta_{1}$ & $\beta_{2}$ & $\beta_{3}$ & $\pi 1$ \\
\hline 0.0214 & 0.0039 & 0.0391 & 1 \\
0.0429 & 0.0079 & 0.0781 & 2 \\
0.0643 & 0.0118 & 0.1172 & 3 \\
0.0857 & 0.0157 & 0.1562 & 4 \\
0.1071 & 0.0197 & 0.1953 & 5 \\
0.1286 & 0.0236 & 0.2344 & 6 \\
\hline
\end{tabular}

Table 3. Average Number of Backorder (ANB) Results on Gold Arrival Rate. Dataset $\left[\mathrm{MST}=6, \mathrm{~S}=10, \mathrm{~K}^{2}=5, \mathrm{~K}^{1}=2, \lambda_{2}=4, \lambda_{3}=3, \mathrm{SL}_{\mathrm{d}}=1, \mathrm{BL}_{\mathrm{d}}=0.12, \mathrm{~L}_{\mathrm{r}}=1\right]$.

\begin{tabular}{cccc}
\hline ANB1 & ANB2 & ANB3 & $\pi 1$ \\
\hline 0.012 & 0.014 & 0.1 & 1 \\
0.024 & 0.028 & 0.2 & 2 \\
0.036 & 0.042 & 0.3 & 3 \\
0.048 & 0.056 & 0.4 & 4 \\
0.06 & 0.07 & 0.5 & 5 \\
0.072 & 0.084 & 0.6 & 6 \\
\hline
\end{tabular}

ANB3. Nine sensitive parameters were perturbed. The nine sensitive parameters are base stock level, gold arrival rate, silver arrival rate, bronze arrival rate, silver demand lead time, bronze demand lead time, first threshold level, second threshold level and replenishment lead time. At each instance of sensitivity analysis, the result of average number of backorders and fill rate of Gold, Silver and Bronze demands were determined and validated by the aid of graphs. 


\section{Conclusions}

PHCN is engaged in over-ordering and this gives a false demand signals as evidenced by eventual returns to the warehouse. This excessive purchasing leads to a backup of parts in inventory. Currently, work/job orders trigger the store's reaction to demand for spare parts. PHCN store unit cannot simply ignore a typical demand. Rather, it needs a better understanding of average number of backorders and the fill rates for all demand classes and how these results relate to the level of risk the company can tolerate. This would be of potential improvement because if the store can understand when it will need parts, then it can plan accordingly to promote just-in-time delivery and minimal inventory. However, work orders are triggers of demand and cause purchase orders to be generated for procurement of parts from the vendors [13].

The research has designed a dynamic algorithm tool that aids decision making and enhances spare parts inventory control and management, which has the capability of performing sensitivity analysis automatically. It can be applied also to supermarkets, pharmaceutical companies, demand and supply organization etc. The major motivation for this research is an experience with the spare parts complex of PHCN. The system does not observe service differentiation through rationing and demand lead time and cannot find the average number of backorders and the fill rates for each demand class, especially manually. It no longer withstands the challenges of modern standards of spare parts inventory control. Therefore, the model presented has formulated, designed and developed a simulation model for effective spare parts inventory which captured the stochastic demands and stepwise state transitions.

\section{References}

[1] Tsakatikas, D. and Rooij, P.J. (2008) Spare Parts Criticality for Unplanned Maintenance of Industrial Systems. European Journal of Industrial Engineering, 2.

[2] Power, D.J. and Ramesh, S. (2007) Model-Driven Decision Support Systems: Concepts and Research Directions. Decision Support Systems, 43, 1044-1061.

[3] Oluwole, A.O. (2012) Inventory and Inventory Control in a Small Business. Management Science, 46, 64-65.

[4] Onohaebi, O.S. (2009) Power Outages in the Nigeria Transmission Grid. Research Journal of Applied Sciences, 4, 1-9.

[5] Batarda, C. (2008) Development of a Power Plant Simulation Model. Instituto Superior Tecnico, Lisbon.

[6] Arobieke, O., Osafehinti, S., Oluwajobi, F. and Oni, O. (2012) Electrical Power Outage in Nigeria: History, Causes and Possible Solutions. Journal of Energy Technologies and Policy, 2, 18-23.

[7] Samuel, I., Katende, J., Daramola, S.A. and Awelala, A. (2014) Review of System Collapse Incidence on 330-kV Nigeria National Grid. International Journal of Engineering Science Invention, 3, 55-59.

[8] Ogbuefi, C.C., Ugwu, C.L. and Ogbogu, N.O. (2018) Analysis of Nigeria Power System Voltage Collapse Incidences from 2000 to 2017. IOSR Journal of Electrical and 
Electronic Engineering (IOSR-JEEE), 13, 28-34.

[9] McHaney, R. (2012) Stochastic Simulation Model: A Real World Processing. Postnet Workshop.

[10] Kranenburg, T. (2008) Pooling in Multi-Location Periodic Inventory Distribution Systems. Omega International Journal of Management Science, 27, 39-59.

[11] Deshpande, V., Cohen, M.A. and Donohne, K. (2003) A Threshold Inventory Rationing Policy for Service-Differentiated Demand Classes. Management Science, 49, 683-703. https://doi.org/10.1287/mnsc.49.6.683.16022

[12] Loiut, K. and Wright R.L.D. (2009) Understanding Statistics. Harcourt Brace Jovanovich Inc., USA.

[13] Dekker, R., Klein, M.J. and Rooij, P.J. (1998) A Spare Parts Stocking System Based on Equipment Criticality. International Journal of Production Economics, 56, 69-77. https://doi.org/10.1016/S0925-5273(97)00050-9

[14] Scala, O. and McHaney, R. (2009) Understanding Computer Simulation, Ventus Publishing Aps., 1-220. 